\title{
Drawings of energy: Evidence of the Next Generation Science Standards model of energy in diagrams
}

\author{
Kara E. Gray, ${ }^{1, *}$ Michael C. Wittmann, ${ }^{2}$ Stamatis Vokos, ${ }^{3}$ and Rachel E. Scherr ${ }^{4}$ \\ ${ }^{1}$ Department of Physics, Seattle Pacific University, \\ 3307 Third Avenue West, Suite 307, Seattle, Washington 98119-1957, USA \\ ${ }^{2}$ Department of Physics, University of Maine, Orono, Maine 04469-5709, USA \\ ${ }^{3}$ Department of Physics, California Polytechnic State University, San Luis Obispo, California 93407, USA \\ ${ }^{4}$ Department of Physical Sciences, University of Washington Bothell, \\ Bothell, Washington 98011-8246, USA
}

(Received 1 November 2018; published 17 May 2019)

\begin{abstract}
The Next Generation Science Standards (NGSS) provide a succession of objectives for energy learning and set an expectation for teachers to assess learners' representations of energy in a variety of science contexts. To support teachers in evaluating the extent to which representations of energy display NGSS objectives, we have (i) discerned the constituent ideas that comprise the NGSS model of energy in the physical sciences and (ii) developed a checklist for assessing the extent to which an energy diagram provides evidence of the NGSS energy model. This energy diagram checklist is representation independent (so that diverse diagrams in a course may all be evaluated) and scenario independent (so that it can be applied throughout the physical science curriculum). We demonstrate the use of the checklist for assessing both pedagogical energy diagrams and learner-invented energy diagrams, including measuring a class's increased facility with energy diagrams.
\end{abstract}

DOI: 10.1103/PhysRevPhysEducRes.15.010129

\section{INTRODUCTION}

Modeling is at the heart of doing science [1,2]. For this work, we take the perspective that models are "explicit representations of a phenomenon that bring certain features into focus" [3]. Scientists use representations to learn abstract scientific concepts and to communicate their understanding of these concepts to others $[1,4]$. The scientific community has created many consensus representations in which scientists are expected to be proficient. Scientists are also expected to invent their own representations to communicate their new thinking. We also take the perspective (attributed to the statistician George Box [5]) that all models are wrong, but some are useful, in the sense that every representation of abstract concepts that are relevant to a particular phenomenon implicitly or explicitly involves a choice of what should be brought to the fore and what can be safely ignored.

Modeling is an important but difficult skill for science learners to develop [6,7]. The Next Generation Science Standards (NGSS) [8] reflects the importance of

\footnotetext{
"grayk5@spu.edu
}

Published by the American Physical Society under the terms of the Creative Commons Attribution 4.0 International license. Further distribution of this work must maintain attribution to the author(s) and the published article's title, journal citation, and DOI. modeling for both doing science and learning science by listing it as one of the eight scientific practices. The NGSS calls for students to develop competency in building and applying "models," [3]. In addition, the NGSS calls for students to learn scientific practices, including model building, within a content context [8]. The NGSS speaks to this through the idea of three dimensional learning which holds that scientific and engineering practices, cross-cutting concepts, and disciplinary core ideas should be intertwined within a lesson or unit and not taught or assessed as independent objectives [3]. For most students, a visual diagram is an important accompaniment to a narrative explanation: the scientific practice of developing models supports the scientific practice of constructing explanations [9].

Energy is an ideal disciplinary core idea in which to ground modeling instruction. Energy is an abstract scientific concept that is shared by all scientific disciplines and is therefore treated by the NGSS as both a disciplinary core idea and as a cross-cutting concept connecting the different sciences [8]. Despite (or perhaps because of) its centrality to scientific thinking, the scientific community does not have a consensus representation of energy for students to use as they learn to apply energy to the various physical, biological, chemical, and earth and space science phenomena that they may encounter [10-14]. Even within the physical sciences, there is no consensus representation for energy - unlike forces, for which a free-body diagram is 
the consensus representational scheme, ${ }^{1}$ or optics, with its light ray diagrams. ${ }^{2}$ The lack of a consensus model can be an obstacle to learning about energy. However, it can also be an opportunity for students to practice inventing representations. Such invented models allow students to "articulate their own understanding of how a scientific phenomenon behaves" [9] clarifying the salient features of the concept and their relationship to each other [15].

However, when learners use diagrams they invent themselves, assessing their representations can be difficult. Teachers need to be prepared to discern the important features of an unfamiliar diagram type and then compare those features to an expected model of energy. This kind of comparison is especially challenging if the expected model of energy is unclear. While the NGSS discusses energy extensively, the model of energy used in the NGSS is not articulated in a straightforward way. Instead, the NGSS's model of energy is presented in a series of complex statements spread throughout the standards. This presents a challenge for teachers who are expected to assess representations of their students' understanding of this concept. In this paper, we focus on teachers assessing students from a formative assessment perspective [16,17]. Within this teaching perspective, teachers are continuously collecting information about students' current understanding and abilities and then using this information to make informed instructional decisions [18-20]. We are less focused on assessment from a summative assessment perspective in which student work is evaluated for the purpose of assigning a grade.

In what follows, we present a clear articulation of the NGSS model of energy in the physical sciences, as we have inferred it, and an associated checklist for energy representations that is independent of a specific representation. Because the checklist is representation independent, it can be used to evaluate diverse learner-invented diagrams. The checklist is also scenario independent, so that it can be applied throughout the physical science curriculum. We argue that certain types of energy representations provide more evidence of the NGSS model of energy, as measured using the presented checklist, and that learners can learn to provide more evidence of the NGSS model of energy in

\footnotetext{
${ }^{1}$ All free-body diagrams represent forces as arrows, with the size of the arrow representing the magnitude of the force. Only forces exerted on the object of interest appear; the net force is the vector sum of all the forces exerted on the object. There are, however, various conventions for labeling individual forces.

${ }^{2}$ Ray diagrams represent the possible paths that light can take to get from one place to another, often from a light source to an observer or screen. Light paths are represented as straight arrows, with the direction of the arrow representing the direction that light travels. Objects (often mirrors, lenses, or screens) are typically shown in cross section. Ray diagrams for curved mirrors and lenses may feature (or may only include) geometrically distinctive rays, such as those that run parallel to the object's axis or through its center.
}

their representations. This ability to provide evidence of a model of energy through a representation is an important part of learning in that representations (i) allow learners to make their thinking about abstract concepts explicit, and (ii) provide teachers an opportunity to interact with reifications [21] of learners' abstract ideas.

In this work, we focus on contexts in which the instructional task is to develop a model of energy. In this context, the goal for considering a particular physical phenomenon is to describe that phenomenon using energy ideas so that both the phenomenon and the model of energy can be better understood. With this learning goal in mind, we take the position that energy diagrams should be explicit about as many features of the energy model as possible. This would not necessarily be the case in the context of a different task. For example, experts using a diagram to tackle an unfamiliar energy problem would not need to make every feature of the energy model explicit in a diagram; in fact, there might be benefit to streamlining their diagrams so that its only explicit features are those relevant to their particular problem. Other features, which are presumably part of the expert's rich and stable model of energy, might remain implicit in an expert's problemsolving diagram. Overall, we recognize that the purpose for which a diagram was created affects whether many or all of the features of the energy model would be explicitly included in the diagram. However, we suggest that energy diagrams created for instructional purposes should ideally include most or all of the features of the energy model.

In Sec. II, we describe the NGSS model of energy in the physical sciences. In Sec. III, we propose a checklist for assessing energy diagrams based on the NGSS model of energy, and apply the checklist to consider how several familiar representations of energy show features of the NGSS model of energy. Section IV presents an assessment of learner-invented diagrams using the checklist. In Sec. V, we use the checklist to demonstrate a class's increased facility with energy diagrams.

\section{NGSS MODEL OF ENERGY IN THE PHYSICAL SCIENCES}

The Next Generation Science Standards [8] and its parent document, the Framework for K-12 Science Education [3], feature energy in both the cross-cutting concepts ("Energy and matter: Flows, cycles, and conservation") and the disciplinary core ideas (including Physical science topic 3, referred to hereafter as "PS3"). These documents do not take the approach of describing their model for energy in a succinct series of statements. Rather, they list a number of standards for understanding energy to be met at three different grade levels (4th grade, middle school, and high school). Most of these standards refer to multiple energy ideas in a single sentence: for example, one of the fourth-grade standards says, "Energy can be moved from place to place by moving objects or 
through sound, light, or electrical currents" (4-PS3.A). The content of different standards overlaps, especially in the case of successive standards: i.e., standards for higher grades add nuance and complexity to those for lower grades. For example, a high school standard that elaborates on the above-cited fourth-grade standard reads, "Energy is a quantitative property of a system that depends on the motion and interactions of matter and radiation within that system. That there is a single quantity called energy is due to the fact that a system's total energy is conserved, even as, within the system, energy is continually transferred from one object to another and between its various possible forms" (HS-PS3.B). These standards provide a succession of learning objectives, which (in the NGSS) are explicitly associated with performance expectations such as, "Apply scientific principles to design, construct, and test a device that either minimizes or maximizes thermal energy transfer" (MS-PS3-3).

In order to develop a checklist for energy representations, we attempted to discern the constituent ideas that comprise the NGSS model of energy in the physical sciences. This approach establishes substantive validity (sound theoretical basis) and content validity (comprehensive coverage) for the checklist [22-24]. We accomplished this discernment of constituent ideas by (i) making a complete list of every energy standard in the cross-cutting concepts and physical sciences sections of the NGSS and the framework, and (ii) identifying the constituent ideas in each standard. To identify the constituent ideas in each standard, we divided the standard into its component semantic parts and associated each part with one or more ideas about energy that we considered to be fundamental features of an energy model. In many cases, such associations are indicated by key words in the standard; in other cases, associations are indicated by phrases. For example, the high-school standard cited above includes the words "system," "conserved," "transferred," and "forms," which are good candidates for constituent ideas. It also includes phrases describing transformation ("between its possible forms") and tracking energy by following its course or trail through a system ("within the system, energy is continually transferred from one object to another"). For another example, the fourth-grade standard cited above includes concepts of energy transfer ("energy can be moved from place to place") and mechanisms of energy transfer ("through sound, light, or electrical currents").

Initially, we identified constituent ideas of the energy model based on our substantial experience with energy model development [12,25-35]. As our analysis continued, we refined our list of constituent ideas to reflect the features of the energy model that appeared most clearly or repeatedly in the standards that we analyzed. In conducting this analysis, we deemphasized references to specific energy scenarios (such as collisions, solar cells, and circuits), in favor of statements contributing to the NGSS model of energy in the physical sciences as a whole. For example, we identify the standard "Energy can be moved from place to place by moving objects or through sound, light, or electrical currents" (4-PS3.A) as conveying the constituent ideas of "transfer" and "mechanism"; we see the specific mechanisms referenced (moving objects, sound, light, and electrical currents) as examples of particular mechanisms, and do not include them among the model's constituent ideas.

Through iterative analysis we identified eleven constituent ideas that we see as comprising the NGSS model of energy in the physical sciences. Table I lists these constituent ideas. Table II documents all the energy standards (exactly as they appear) in NGSS physical science topic 3 and the constituent ideas associated with each standard. Table III documents the constituent ideas associated with each (verbatim) statement about energy in the relevant cross-cutting concept. To further establish the validity of the constituent ideas, we obtained expert review: six experts in the teaching and learning of energy in physics

TABLE I. Eleven constituent ideas comprising the NGSS model of energy in the physical sciences.

\begin{tabular}{ll}
\hline \hline Term & \\
\hline Conservation & The number of energy units remains constant in a scenario. \\
Tracking & Energy may be tracked by following its path among objects, fields, and systems. \\
Forms & Energy manifests in multiple forms, e.g., kinetic energy, gravitational energy, and thermal energy. \\
Observables & Forms of energy are indicated ${ }^{\mathrm{a}}$ by observable quantities, e.g., thermal energy is indicated by temperature. \\
Transformation & Energy can change from one form to another. \\
Transfer & Energy can move from one object or field to another. \\
Mechanism & Energy transfer occurs through specific mechanisms or processes, e.g., pushing, conduction, or metabolism. \\
System & A collection of relevant objects in a scenario may be defined by a boundary that energy may cross. \\
Spreading & Uncontrolled systems evolve toward more even energy distribution. \\
Usefulness & Some forms of energy are less useful, e.g., thermal energy in the environment. \\
Mathematization & The amount of energy associated with observable quantities and object properties can be expressed mathematically \\
& (e.g., kinetic energy $=1 / 2 \mathrm{mv}^{2}$ ). \\
\hline \hline
\end{tabular}

\footnotetext{
${ }^{\mathrm{a}}$ We consider an indicator of a form to be that measurable property of the object or system that indicates the presence of a form in a specific object. In this sense, speed is the indicator of kinetic energy. The mass of the object is a factor that allows us to compare kinetic energies of two objects with the same speed.
} 
TABLE II. NGSS energy standards in the disciplinary core ideas (physical science topic 3) and their constituent ideas.

Disciplinary core idea (DCI), Physical science topic 3

The faster a given object is moving, the more energy it possesses. (4-PS3.A)

Energy can be moved from place to place by moving objects or through sound, light, or electrical currents. (4-PS3.A)

Motion energy is properly called kinetic energy; it is proportional to the mass of the moving object and grows with the square of its speed. (MS-PS3.A)

A system of objects may also contain stored (potential) energy, depending on their relative positions. (MS-PS3.A)

Temperature is a measure of the average kinetic energy of particles of matter. The relationship between temperature and the total energy of a system depends on the types, states, and amounts of matter present. (MS-PS3.A)

Energy is a quantitative property of a system that depends on the motion and interactions of matter and radiation within that system. That there is a single quantity called energy is due to the fact that a system's total energy is conserved, even as, within the system, energy is continually transferred from one object to another and between its various possible forms. (HS-PS3.A)

At the macroscopic scale, energy manifests itself in multiple ways, such as in motion, sound, light, and thermal energy. (HS-PS3.A)

These relationships are better understood at the microscopic scale, at which all of the different manifestations of energy can be modeled as a combination of energy associated with the motion of particles and energy associated with the configuration (relative position) of the particles. In some cases, the relative position energy can be thought of as stored in fields (which mediate interactions between particles).... (HS-PS3.A)

Energy is present whenever there are moving objects, sound, light, or heat. When objects collide, energy can be transferred from one object to another, thereby changing their motion. In such collisions, some energy is typically also transferred to the surrounding air; as a result, the air gets heated and sound is produced. (4-PS3.B)

Light also transfers energy from place to place. (4-PS3.B)

Energy can also be transferred from place to place by electrical currents, which can then be used locally to produce motion, sound, heat, or light. The currents may have been produced to begin with by transforming the energy of motion into electrical energy. (4-PS3.B)

When the kinetic energy of an object changes, there is inevitably some other change in energy at the same time. (MS-PS3.B)

The amount of energy transfer needed to change the temperature of a matter sample by a given amount depends on the nature of the matter, the size of the sample, and the environment. (MS-PS3.B)

Energy is spontaneously transferred out of hotter regions or objects and into colder ones. (MS-PS3.B)

Conservation of energy means that the total change of energy in any system is always equal to the total energy transferred into or out of the system. (HS-PS3.B)

Energy cannot be created or destroyed, but it can be transported from one place to another and transferred between systems. (HS-PS3.B)

Mathematical expressions... allow the concept of conservation of energy to be used to predict and describe system behavior. (HS-PS3.B)

The availability of energy limits what can occur in any system. (HS-PS3.B)

Uncontrolled systems always evolve toward more stable states - that is, toward more uniform energy distribution (e.g., water flows downhill, objects hotter than their surrounding environment cool down). (HS-PS3.B)

When objects collide, the contact forces transfer energy so as to change the objects' motions. (4-PS3.C)

When two objects interact, each one exerts a force on the other that can cause energy to be transferred to or from the object. (MS-PS3.C)

When two objects interacting through a field change relative position, the energy stored in the field is changed. (HS-PS3.C)

The expression "produce energy" typically refers to the conversion of stored energy into a desired form for practical use. (4-PS3.D)
Constituent ideas

Observables

Transfer mechanism

Observables mathematization

System forms observables

Observables

System conservation transfer forms transformation

Forms

Forms

Observables transfer mechanism spreading

Transfer

Transfer forms transformation

Transfer conservation $^{\mathrm{a}}$

Mathematization transfer observables

Transfer spreading

Conservation transfer system

Conservation transfer system

Mathematization system

Usefulness system

System spreading

Transfer observables mechanism

Transfer mechanism

Transformation mechanism observables

Transformation forms usefulness 
TABLE II. (Continued)

\begin{tabular}{lc}
\hline \hline Disciplinary core idea (DCI), Physical science topic 3 & Constituent ideas \\
\hline $\begin{array}{l}\text { Although energy cannot be destroyed, it can be converted to less useful forms-for example, to } \\
\text { thermal energy in the surrounding environment. (HS-PS3.D) }\end{array}$ & $\begin{array}{c}\text { Conservation forms transformation } \\
\text { usefulness }\end{array}$ \\
\hline \hline
\end{tabular}

${ }^{\text {a }}$ Standard MS-PS3.B appears in a section exclusively concerned with energy transfer and conservation, though out of context it could also seem to refer to energy transformations.

TABLE III. NGSS energy statements in the cross-cutting concepts (energy and matter) and their constituent ideas.

\begin{tabular}{|c|c|}
\hline Cross-cutting concept (CCC), Energy and matter; Flows, Cycles, and Conservation & Constituent ideas \\
\hline Energy can change only through transfers into or out of the system. & $\begin{array}{l}\text { Transfer system } \\
\text { conservation }\end{array}$ \\
\hline It is very informative to track the transfers of energy within, into, or out of any system under study. & $\begin{array}{l}\text { Transfer system } \\
\text { tracking }^{\mathrm{a}}\end{array}$ \\
\hline $\begin{array}{l}\text { Any cycle of matter also involves associated energy transfers at each stage, so to fully understand the water } \\
\text { cycle, one must model not only how water moves between parts of the system but also the energy transfer } \\
\text { mechanisms that are critical for that motion. }\end{array}$ & $\begin{array}{l}\text { Transfer mechanisms } \\
\text { tracking }\end{array}$ \\
\hline Consideration of energy inputs, outputs, and flows or transfers within a system or process are important. & $\begin{array}{l}\text { Transfer system } \\
\text { tracking }\end{array}$ \\
\hline $\begin{array}{l}\text { The ability to examine, characterize, and model the transfers and cycles of energy is a tool that students can } \\
\text { use across virtually all areas of science and engineering. }\end{array}$ & Transfer tracking \\
\hline
\end{tabular}

\footnotetext{
${ }^{\text {a }}$ Tracking is positioned in the framework as central to the cross-cutting concept of energy. Therefore, even though tracking is not mentioned in the DCIs, we consider it to be an important part of the NGSS's overall model of energy and on equal footing with the other constituent ideas of the model that appear frequently in the DCIs (such as forms or transfer).
}

commented in detail on our initial drafts of Tables I-III, resulting in specific changes to the constituent ideas (for example, the term "mathematization" replaced the former term "quantification" to emphasize the use of mathematical expressions). Future work will expand on this validation work by studying teachers' use of the checklist with their own students.

The NGSS model of energy in the physical sciences consistently employs a metaphor in which energy is a substance and objects and fields are containers for that substance. For example, objects "possess" energy (4PS3.A), a system "contains" energy (MS-PS3.A), energy is "stored in fields" and "moves across space" (HS-PS3.A). The NGSS's use of a substance metaphor for energy is consistent with its emphasis on energy conservation, transfer, and flow [11,36-41] and with how physicists conceptualize energy [42], and is typical in classical mechanics [42]. Other models of energy, based on a metaphor in which energy is a vertical location, may be preferable in contexts that require negative energies [43] or refer to energy levels, as in atomic and quantum physics $[12,42]$.

\section{DEVELOPMENT AND APPLICATION OF A CHECKLIST FOR ASSESSING ENERGY DIAGRAMS}

\section{A. Energy diagram checklist}

We use the eleven constituent ideas about energy listed in Table I to evaluate the extent to which an energy diagram instantiates the NGSS model of energy. For each constituent idea, we identify how that idea might be explicitly represented in a diagram. For example, the idea of conservation may be represented by picturing energy units explicitly and showing the same number of energy units as being present throughout a scenario; the idea of tracking may be indicated by following the course or trail of a unit of energy, noting its form and location at various points as well as the transfers and transformations it experiences as the scenario unfolds. Table IV presents how each constituent idea may show in an energy diagram. This is the energy diagram checklist. (The checklist does not include energy usefulness [25] or mathematization because we are not aware of a means by which these ideas could be represented in a diagram.) Validation and reliability of the checklist are discussed in Sec. III. D.

This checklist does not assess a learner's energy model; rather, it assesses a diagram, which we interpret as a learners' use of a specific representation of energy in a specific scenario. For example, learners using bar charts are not likely to display their understanding of energy tracking, because that representation does not foreground that feature of the energy model; this absence of tracking in the representation implies nothing about the learners' understanding of energy tracking. Similarly, a learner analyzing a scenario involving only a single object will not be able to demonstrate understanding of energy transfers. The checklist may help instructors to identify which features of the energy model are foregrounded in particular representations. 
TABLE IV. Checklist for assessing the extent to which an energy diagram shows constituent ideas of the NGSS model of energy.

\begin{tabular}{|c|c|}
\hline Constituent idea & How it might show in a diagram \\
\hline Conservation & $\begin{array}{l}\text { Quantities or units of energy are pictured explicitly and same quantity of energy or number of energy units are } \\
\text { visible throughout scenario }\end{array}$ \\
\hline Tracking & $\begin{array}{l}\text { Quantities or units of energy are pictured explicitly and each quantity or unit of energy comes from and/or goes to } \\
\text { somewhere and each quantity or unit of energy experiences a series of transfers and/or transformations }\end{array}$ \\
\hline Forms & Specific types of energy are pictured, e.g., motion energy, elastic energy, thermal energy \\
\hline Observables & $\begin{array}{l}\text { Specific types of energy are pictured and observable quantities are associated with each type of energy, e.g., warmth } \\
\text { or temperature with thermal energy }\end{array}$ \\
\hline Transformation & $\begin{array}{l}\text { Quantities or units of energy are pictured explicitly and quantities or units of energy are shown changing from one } \\
\text { form to another }\end{array}$ \\
\hline Transfer & $\begin{array}{l}\text { Quantities or units of energy are pictured explicitly and quantities or units of energy are located in objects, fields, or } \\
\text { systems and quantities or units of energy are shown moving from one object or field to another }\end{array}$ \\
\hline Mechanism & $\begin{array}{l}\text { Quantities or units of energy are shown moving from one object or field to another and the means by which energy } \\
\text { moves from place to place, e.g., conduction or shoving, is labeled (or the means by which energy transforms, } \\
\text { e.g., metabolism or compression, is labeled) }\end{array}$ \\
\hline System & $\begin{array}{l}\text { Quantities or units of energy are located in objects, fields, or systems and a boundary is indicated that energy might } \\
\text { cross, such that some energy is inside and some is possibly outside }\end{array}$ \\
\hline Spreading & Energy spreads to more objects (or energy goes to large objects, e.g., the air or the environment) \\
\hline Usefulness & (Not part of a diagram) \\
\hline Mathematization & (Not part of a diagram) \\
\hline
\end{tabular}

\section{B. Perspectives inherent in the use of checklists}

To use a checklist is to subscribe, perhaps implicitly, to a number of assumptions. In particular, checklists embody the perspective that a complex idea (in this case, the NGSS model of energy in the physical sciences) may be expressed in terms of a number of discrete elements (in this case, the constituent ideas about energy listed in Table IV). As a consequence, an energy diagram checklist may create the expectation that energy diagrams can be easily compared with each other: for example, that one diagram is twice as good as another if it includes twice as many constituent ideas. We do take the position that roughly speaking, the extent to which a diagram shows the NGSS model of energy is indicated by the number of checklist items that are satisfied by the diagram. In other words, the greater the number of NGSS constituent ideas represented, the "better" the diagram (if "better" means "more completely displaying the NGSS model of energy"). However, it is inappropriate to add scores on individual constituent ideas to obtain an overall score (e.g., to give a diagram a score of 6/9 if it demonstrates six of the nine constituent ideas in the checklist), since the items in the energy diagram checklist are neither independent nor of equal weight. This means that we also cannot say that a diagram which includes eight constituent ideas from the checklist is definitively better than one that shows seven, or that two diagrams that each show seven constituent ideas are equal.

The fact that we cannot reliably interpret a "score" on the energy diagram checklist, or use such a score to quantitatively compare diagrams, has to do with the binary nature of the checklist. Checklist items may be assessed as if they were either present or not present (a diagram either shows conservation of energy, or does not show conservation of energy) even though in some cases there may be differences of degree (some diagrams may show energy conservation more strongly than others) or of kind (some diagrams may show energy conservation in a different way than others). It might be possible to expand our checklist into a rubric that evaluates the degree to which each constituent idea is present, as in a learning progressions approach [44]. However, a learning progressions approach aims to create a hierarchy of increasingly sophisticated ideas about a concept, imagining that learners will progress through this hierarchy to ultimately arrive at a more complete and correct idea [9]. In contrast, we do not imagine a hierarchy of energy representations. Rather, we believe that there may be a variety of different energy diagrams that all represent the NGSS model of energy but are suited to different instructional settings or purposes.

What the checklist does do is show whether a given feature of the energy model is explicit and therefore visible to learners in a given diagram. From a pedagogical perspective, the checklist suggests which constituent ideas of the NGSS model a learner is comfortable working with and which constituent ideas they consider relevant to a given scenario. For constituent ideas of the NGSS model of energy that are not included in the diagram, an instructor may consider why not. For example, it may be that the physical scenario being represented did not offer learners the opportunity to demonstrate that constituent idea; if a scenario only includes heat transfer between two objects, the related diagram will not include transformations. Alternatively, it may be that the learner has left that constituent idea of the NGSS model of energy implicit, or that constituent idea of the NGSS model of energy is not a part of the learners' understanding of energy: a diagram 


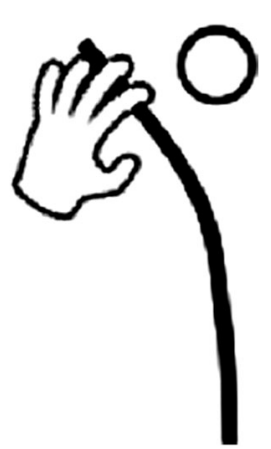

FIG. 1. Top view of ring slider scenario, in which a metal ring is smacked by a bent-back ruler and slides across the floor.

that does not show conservation may suggest that the learner does not consider energy conservation to be relevant to the current scenario, or that they do not consider energy to be a conserved quantity. When considering multiple diagrams, it can also be pedagogically effective to consider which constituent ideas both diagrams include and to discuss with learners whether the diagrams represent that constituent ideas in the same way or if they use different representation strategies. Learners can then consider if one strategy communicates this constituent ideas more effectively or efficiently. Such an exercise would use the information from the checklist as a starting point for pedagogical activity.

From a research perspective, a checklist allows us to consider which diagrams make more constituent ideas of the NGSS model of energy explicit and which diagrams leave constituent ideas as implicit or do not include those ideas in their implied model of energy. Diagrams with more constituent ideas help learners more easily visualize or communicate the NGSS model of energy as they are learning about energy. Therefore, the checklist helps us to select diagrams for pedagogical purposes or to consider learners' ability to communicate their thinking about energy in light of the NGSS model of energy.

\section{Examples of pedagogical energy diagrams}

To illustrate the use of the checklist, we assess three pedagogical diagrams about energy (diagrams that appear in textbooks or other published instructional materials): a pair of energy bar charts, an energy flow diagram, and an energy tracking diagram [28]. We analyze the bar charts and the energy tracking diagram in the context of a "ring slider" scenario, in which a metal ring is smacked by a bentback ruler and slides across the floor (Fig. 1). The diagrams show the energy of the ruler, ring, and environment (floor and air). The energy flow diagram depicts a generalized engine scenario. Table $\mathrm{V}$ summarizes the constituent ideas of the NGSS model of energy represented in these pedagogical energy diagrams.

\section{Bar chart}

A pair of bar charts for the ring slider scenario is shown in Fig. 2. The first chart depicts the energy when the ruler is bent back, before it smacks the ring; the second chart depicts the energy at a later moment, while the ring is sliding and the ruler is no longer bent.

To assess a diagram according to the energy diagram checklist, we score each constituent idea by whether that idea is explicitly shown in the diagram. The pair of bar charts in Fig. 2 depicts two constituent ideas of the NGSS model of energy: Energy is conserved in that the height of the "total energy" bar is the same in both charts, and multiple forms of energy are depicted (kinetic, potential, and thermal).

Several other constituent ideas of the NGSS energy model, however, are not shown in the bar charts in Fig. 2. Energy is not tracked in its course through different objects in the scenario, because no objects are depicted. Energy is not shown transferring from one object to another. Mechanisms of energy transfer are not indicated. Types of energy are not associated with observable quantities, i.e., thermal energy with temperature. Energy units are not explicitly shown as transforming from one form to another (though one might infer such transformation since the distribution of energy among various forms is shown to change). Energy is not shown as spreading among objects or in space, because no objects are depicted. Finally, a system is not depicted, in that there is no boundary indicated that energy might cross. (We might infer that the system is all the objects whose energy is included in the diagram - the ruler, the ring, and the environment - but this would be an inference; the system might equally well contain only the ruler and the ring, or only the ring.) Overall, the bar charts in Fig. 2 do not show many constituent ideas of the NGSS model of energy. Some instructors and researchers use bar charts with

TABLE V. Summary of constituent ideas of the NGSS model of energy represented in three pedagogical energy diagrams.

\begin{tabular}{lccccccccc}
\hline & Conservation & Tracking & Forms & Observables & Transformation & Transfer & Mechanism Systems & Spreading \\
\hline Bar chart & $\mathrm{X}$ & & $\mathrm{X}$ & & $(\mathrm{X})^{\mathrm{a}}$ & & & & \\
Energy Flow Diagram & $\mathrm{X}$ & $\mathrm{X}$ & & & $\mathrm{X}$ & $\mathrm{X}$ & $\mathrm{X}$ & $\mathrm{X}$ \\
Energy Tracking Diagram & $\mathrm{X}$ & $\mathrm{X}$ & $\mathrm{X}$ & $(\mathrm{X})$ & $\mathrm{X}$ & $\mathrm{X}$ & $\mathrm{X}$ & $(\mathrm{X})$ & $\mathrm{X}$ \\
\hline \hline
\end{tabular}

${ }^{a}$ Consituent ideas in parentheses are not typically explicit in that type of diagram, but could be inferred from the diagram or could be included if the diagram was modified slightly. 


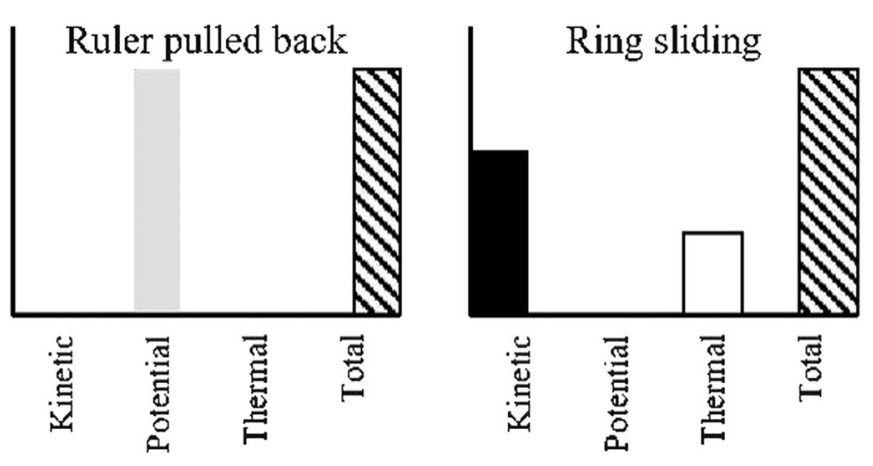

FIG. 2. Energy bar charts for ring slider scenario. While the ruler is pulled back (before it smacks the ring), all of the energy is potential energy. While the ring is sliding, the energy is partly kinetic (the ring is moving) and partly thermal (the ring and floor warm as they rub together).

additional features, such as explicitly representing systems [45] or work [10]; such diagrams show more constituent ideas of the NGSS model of energy.

\section{Energy flow diagram}

Energy flow diagrams are commonly used in the study of thermodynamics to represent the conversion of heat into work in heat engines and other devices. Figure 3 shows the energy flow for any continuously operating reversible device generating work from heat [46]. (The ring slider is not such a device.) This type of diagram depicts heat from a high-temperature reservoir $\left(Q_{h}\right)$ being input into a system (shown on the diagram as a gray box); some of this heat is converted into work $(W)$ that leaves the system, and some of the heat flows through the system to be output to a lowtemperature reservoir $\left(Q_{l}\right)$. The quantity of energy flow is represented by the width of the arrows.

The diagram in Fig. 3 depicts many constituent ideas of the NGSS model of energy. Energy is shown as being conserved in that the total width of the arrows is constant throughout the process. A system is explicitly shown (gray box), with energy crossing the boundaries of the system.

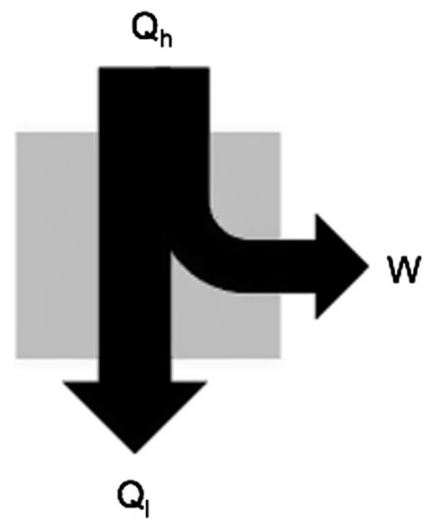

FIG. 3. Energy flow diagram for any continuously operating reversible device generating work from heat.
Energy is tracked in its course through the scenario; though the objects among which it flows are not made visible, the diagram shows transfer from one object to another (e.g., from the high-temperature reservoir to the low-temperature reservoir) as well as mechanisms of transfer (e.g., heat and work). The diagram also shows energy spreading, in that energy that started out in a single place is distributed to two places in the course of the scenario (one to the right of the gray box, the other below the gray box).

Other constituent ideas of the NGSS model of energy are not depicted in Fig. 3. Forms of energy (such as thermal energy) are not shown; likewise, the diagram does not show transformations of energy (e.g., from thermal to kinetic), or associate energy forms with observable indicators such as temperature. Finally, this type of energy flow diagram focuses almost exclusively on the industrial conversion of heat to work, rather than supporting analysis of energy as a cross-cutting concept in physical, biological, chemical, and earth science scenarios [8]. Overall, though, energy flow diagrams of the type modeled in Fig. 3 show many constituent ideas of the NGSS model of energy.

\section{Energy tracking diagram}

In an energy tracking diagram [28], objects are represented as schematic areas and individual energy units are represented as letters, with the specific letter representing the form of energy. Energy transfers and transformations are represented with arrows. The process or mechanism by which a transfer or transformation occurs (e.g., mechanical work) is represented by the color or pattern of the arrow. Relative quantities of energy may be represented by adding coefficients to the letters that represent units of energy.

Figure 4 is an energy tracking diagram for the ring slider scenario. In this scenario, elastic energy in the ruler transforms into kinetic energy (the ruler moves), which transfers to the ring through mechanical work. That kinetic energy is then transformed into thermal energy in the ring and the floor as the ring slides across the floor. In the diagram, $E, K$, and $T$ represent elastic, kinetic, and thermal energy; black, gray, and white arrows represent elastic expansion, mechanical work, and dissipation, respectively.

Energy tracking diagrams display many constituent ideas of the NGSS model of energy. They show energy being conserved in that units of energy are pictured explicitly as

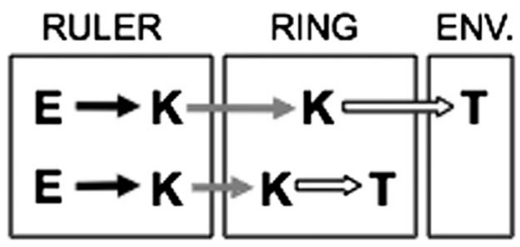

FIG. 4. Energy tracking diagram for ring slider scenario. $E, K$, and $T$ represent elastic, kinetic, and thermal energy. Black, gray, and white arrows represent elastic expansion, mechanical work, and dissipation. 
persisting throughout the transfers and transformations that comprise the scenario. (The scenario pictured in Fig. 4 begins and ends with two units of energy, found by counting the number of chains of arrows.) Energy is tracked by following its path through different objects, with energy transfers explicitly associated with specific mechanisms and processes. There are multiple forms of energy, and energy transformations are shown explicitly. Finally, energy is shown as spreading, if we are willing to assume that by the environment being physically large there are more ways in which energy can spread.

Energy tracking diagrams may be optionally modified to indicate a specific system by drawing a boundary around the objects to be included in the system. (For example, a boundary could enclose the ruler and ring together, or only the ruler.) Energy tracking diagrams do not typically include the observable quantities associated with specific forms of energy, but could be optionally modified to include a supplementary chart with a list of forms and corresponding observables. (Other diagrams could also be supplemented in this way.) Overall, energy tracking diagrams show most of the constituent ideas of the NGSS model of energy and could be modified to show those not usually included.

The above analysis indicates that the checklist distinguishes between pedagogical energy diagrams according to the number of constituent ideas of the NGSS model of energy that are shown in each diagram. While bar charts are frequently used to represent energy, the type considered in Fig. 2 leaves many of the constituent ideas of the NGSS model of energy implicit. Energy flow diagrams explicitly show more constituent ideas of the NGSS model of energy but are only applicable to certain types of scenarios. Energy tracking diagrams show most, though not all, of the constituent ideas of the NGSS model of energy and can be applied in a wide variety of scenarios.

\section{Validation and reliability}

The energy diagram checklist is intended to assess the extent to which diagrams exhibit the NGSS energy model in the context of a particular scenario. The validity of this checklist for assessing pedagogical energy diagrams or learners' energy diagrams rests on its tight alignment with the NGSS and on expert review, as described in Sec. II. The reliability of the energy diagram checklist is established through tests of interrater reliability. Reliability data consist of diagrams from courses in which learners individually produced an energy diagram for a particular scenario: in other words, learners responded in writing to a question such as, "Draw a diagram showing the energy transfers and transformations within and/or among the objects in the given scenario."

The data whose reliability we analyzed consist of diagrams produced by $\mathrm{K}-12$ teachers in professional development courses on the teaching and learning of energy. Teachers take these courses because they feel unprepared to teach about energy. Their diagrams show substantial variety, and because they are expert learners with professional motivations, they are articulate and thorough in their responses. For these reasons, K-12 teachers are an excellent study population-in many ways preferable to a population of younger learners, especially in the exploratory phases of a project.

We applied the checklist to diagrams from three different teacher professional development courses (two for elementary teachers and one for middle school teachers) in two different years (2014 and 2015) at three different universities (Seattle Pacific University, University of Maine, and Tufts University). These data included diagrams of seven different mechanical scenarios (a rubber-band-powered propeller, a ring slider, a penguin walking uphill, a ball rolling downhill into a cup, a plastic popper toy, a solar panel, and a windmill). Each diagram was evaluated for the presence of each of the nine constituent ideas in the checklist, resulting in a large number of opportunities for agreement or disagreement.

Initially, two researchers (K. G. and R. S.) applied the checklist collaboratively to learners' diagrams of one scenario (the propeller) in the two 2015 courses to establish a shared understanding of how to apply the checklist (14 diagrams, each assessed for all 9 constituent ideas, for a total of 126 individual items assessed). These same two researchers then scored all of the remaining 96 diagrams independently and compared assessments of each of the nine individual constituent ideas that potentially appeared in each diagram (e.g., conservation, tracking, forms). This test of interrater reliability was conducted in batches by course and scenario (e.g., all of the diagrams for the 2014 ring slider scenario were scored together). Reliability was assessed according to the following expression:

(No. possible codes for all diagrams in the batch) - (No. disagreements in all diagrams in the batch),

(No. possible codes for all diagrams in the batch),

which may also be written as

(No. constituent ideas $) \times($ No. diagrams in the batch $)-($ No. disagreements in all diagrams in the batch $)$, (No. constituent ideas $) \times($ No. diagrams in the batch $)$. 
TABLE VI. Reliability data for energy diagram checklist.

\begin{tabular}{|c|c|c|c|c|c|c|c|}
\hline Year & Institution & Participants & $\begin{array}{l}\text { No. of } \\
\text { learners }\end{array}$ & No. of scenarios & $\begin{array}{c}\text { No. of } \\
\text { diagrams }\end{array}$ & $\begin{array}{l}\text { No. of potential } \\
\text { agreements }\end{array}$ & IRR \\
\hline 2014 & $\begin{array}{c}\text { University of } \\
\text { Maine }\end{array}$ & $\begin{array}{c}\text { Middle school } \\
\text { teachers }\end{array}$ & 18 & $\begin{array}{c}3 \text { (ring slider, penguin, } \\
\text { ramp-cup) }\end{array}$ & 54 & 486 & $\begin{array}{l}0.82-0.94 \\
\text { (ave. } 0.87 \text { ) }\end{array}$ \\
\hline 2015 & Tufts University & $\begin{array}{c}\text { Elementary school } \\
\text { teachers }\end{array}$ & 6 & $\begin{array}{c}3 \text { (popper, solar panel, } \\
\text { windmill) }\end{array}$ & 18 & 162 & $\begin{array}{l}0.85-0.94 \\
\text { (ave. } 0.90 \text { ) }\end{array}$ \\
\hline 2015 & $\begin{array}{c}\text { Seattle Pacific } \\
\text { University }\end{array}$ & $\begin{array}{c}\text { Elementary school } \\
\text { teachers }\end{array}$ & 8 & $\begin{array}{c}3 \text { (popper, solar panel, } \\
\text { windmill) }\end{array}$ & 24 & 216 & $\begin{array}{l}0.72-0.94 \\
\text { (ave. } 0.83 \text { ) }\end{array}$ \\
\hline Total & & & 32 & 6 & 96 & 864 & \\
\hline
\end{tabular}

The number of constituent ideas is nine. For a course with 15 participants, a batch of diagrams with 10 disagreements would correspond to an interrater reliability of $(9 \times 15-10) /(9 \times 15)=92 \%$.

After each batch of applications of the checklist, scoring decisions were documented in a casebook for consultation in future applications of the checklist. Over 800 individual items were scored. Interrater reliability averaged about 0.88 overall; reliability improved with experience and the development of the casebook. Preliminary coding disagreements were always resolved after discussion. Table VI presents the interrater reliability data. On this basis, we conclude that the checklist is reliable.

\section{ASSESSMENT OF LEARNER-INVENTED ENERGY DIAGRAMS}

A primary benefit of a valid and reliable checklist for assessing energy diagrams is the opportunity to assess original diagrams invented by learners to express their ideas about energy in a physical scenario. In this section, we analyze original learner diagrams to assess the extent to which they show constituent ideas of the NGSS model of energy outlined above. Since the checklist is representation independent, such an assessment tool may support teachers in empowering their students to create representations while also analyzing the diagrams for the constituent ideas of the NGSS model of energy and observing missing constituent ideas. The checklist suggests which learnerinvented diagrams successfully communicate the NGSS model of energy. The checklist also points to diagrams in which constituent ideas of the NGSS model of energy are implicit or even contradicted. Indirectly, the checklist may also point to instructional interventions that might help advance a learner's model of energy: for instance, if a learner-invented diagram does not include tracking, it may be helpful to ask the learner where a particular energy unit in the diagram came from or is going.

The diagrams assessed below were produced by inservice teachers in professional development courses at Seattle Pacific University. The data includes diagrams of four different mechanical scenarios (a wind turbine, a ring slider, a horizontally compressed spring, and a singlebulb circuit).

\section{A. Energy picture}

A learner-invented diagram of the energy in a wind turbine scenario is shown in Fig. 5. A wind turbine is a device for transforming energy from wind to electrical energy. The learner's diagram associates the sun with light, heat, and thermal energy, the wind with motion energy, and the blades of the wind turbine with motion energy. This diagram shows only a few constituent ideas of the NGSS energy model. It represents energy in multiple forms. It represents the observables that indicate those forms by labeling the light and heat from the sun and showing arrows or lines of movement to indicate the observable motion indicative of motion energy.

This diagram foregrounds the forms of energy and not movement of energy among different objects. Because it does not show energy moving from one object to another or transforming from one form to another in the course of the scenario, this diagram does not include most of the NGSS constituent ideas of energy including tracking, conservation, transformation, transfer, mechanism, and spreading. The diagram also does not include an explicit system since no boundary is shown that energy might cross.

\section{B. Energy trajectory}

A learner-invented diagram of the energy in the ring slider is shown in Fig. 6. It shows elastic energy going to kinetic energy in the ruler, then going to kinetic energy in
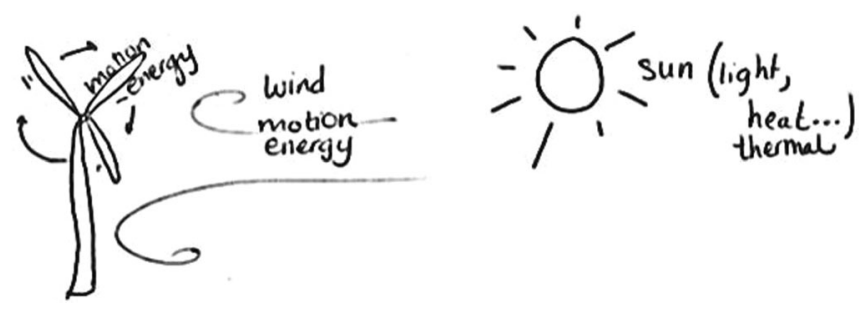

FIG. 5. A learner-invented "energy picture" diagram for a wind turbine scenario. 


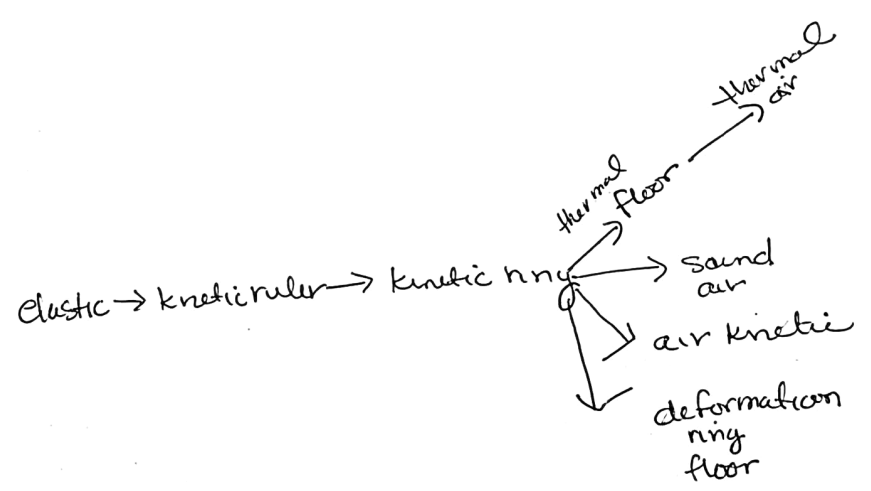

FIG. 6. A learner-invented "energy trajectory" diagram for a ring slider scenario.

the ring. From there the energy goes into the floor and air as thermal, sound, kinetic, and deformation energy.

Unlike the previous diagram (Fig. 5) that emphasized the forms of energy located in certain objects and the observables that indicate each energy form, this diagram emphasizes the movement of energy among objects and forms, and shows energy distributing to more objects and forms as time passes. The diagram is similar in some ways to an energy tracking diagram in that it represents transfers and transformations with arrows, and it includes several constituent ideas of the NGSS energy model: energy is transferred to multiple objects, appears in multiple forms, and transforms from one form to another. The diagram shows the spreading of energy to several objects. The diagram also shows elements of tracking, in that the energy is always shown as coming from somewhere and going somewhere else, though individual units of energy are not pictured.

However, there are several constituent ideas of the NGSS energy model not included in this diagram. The diagram does not show conservation of energy. Because units of energy are not shown, this diagram is not able to show that the amount of energy remains constant even as it is distributed to multiple objects. The diagram does not indicate a system, since no boundary is shown such that energy could be inside or outside a boundary. The diagram does not show mechanisms of energy transfer or transformation: while transfers and transformations are indicated by arrows, they are not labeled. (Though the learner mentions deformation, this seems to be representing a form of energy rather than a mechanism of energy transformation.) The diagram does not mention observables.

\section{Energy source and receiver}

A learner-invented diagram of compression of a horizontal spring is shown in Fig. 7. This diagram is a version of the source-receiver diagrams used in the Physics and Everyday Thinking curriculum [13]. The diagram includes three different energy processes that occur in sequence, with time moving down the page. The rectangles represent
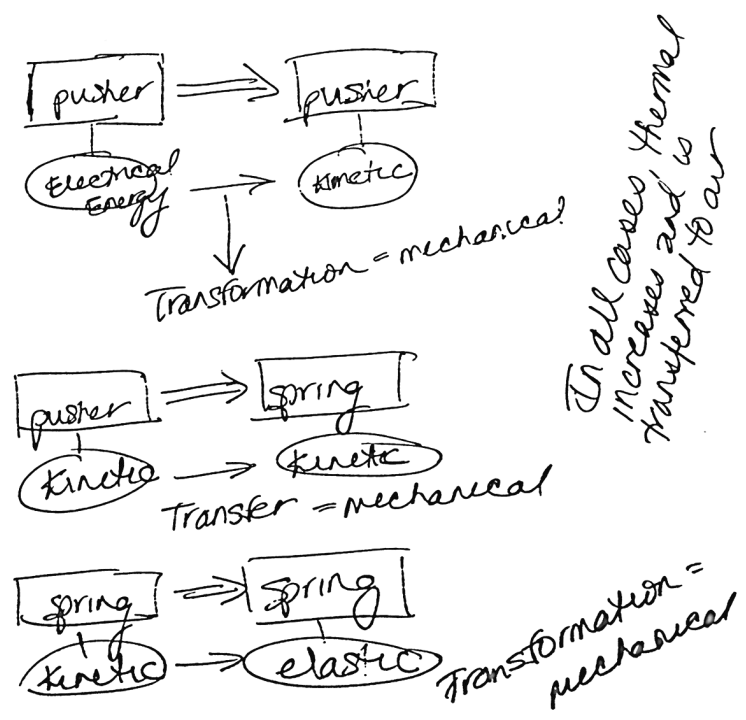

FIG. 7. A learner-invented "energy source and receiver" diagram for a spring compression scenario.

the objects in the scenario, and the form of energy in each object is indicated in the circle connected to that object. The arrows represent energy transfer or transformation and each is labeled with a mechanism. In this scenario, the spring is compressed horizontally by a machine, which the learner calls the "pusher"; the energy in the scenario starts out as electrical energy in the pusher, then transforms to kinetic energy through what the learner calls a mechanical transformation. In the next step, kinetic energy transfers from the pusher to the spring through what the learner calls a mechanical transfer. Finally, the kinetic energy in the spring is transformed to elastic energy through a mechanical transformation. The learner includes a note that thermal energy is increasing throughout the scenario, but thermal energy is not shown in the diagram.

As with the previous diagram, this learner-invented diagram emphasizes the movement of energy between objects and forms. Unlike the previous diagram, this diagram explicitly associates each energy form with a specific object. This representation also provides a way to indicate the mechanism of the transfers and transformations, though in this specific diagram all mechanisms are labeled the same type.

This diagram includes several of the constituent ideas of the NGSS energy model. Energy is tracked as it moves from one object to another (as indicated both by the arrows and by the fact that the right-hand rectangles and circles of one step are identical to the left-hand rectangles and circles of the following step). Forms of energy are shown in the circles of the diagram. Transfers are indicated by a change in the object shown in the rectangle, while transformations are indicated by a change in the energy form shown in the circle. Transfers and transformations are also labeled. The mechanisms for transfer and transformation are also 
labeled, though this diagram shows the same mechanism for all transfers and transformations.

This diagram does not show several constituent ideas of the NGSS energy model. Energy is not explicitly conserved: energy units are not shown and the diagram describes thermal energy as increasing without accounting for a corresponding decrease in energy. No system is indicated since a boundary is not shown. The observables that indicate the presence of each form of energy are not included. Energy spreading is not shown: the energy appears to move from one object to another without spreading out.

\section{Energy snapshots}

Another learner-invented diagram of the energy in a wind turbine is shown in Fig. 8. This diagram shows four objects for the scenario: the air, the blades of the wind turbine, the wind turbine generator, and the wires that carry electricity away from the wind turbine. The objects and their energy units are shown at successive moments in time with time moving down the diagram. The energy units are represented by individual letters within the labeled circles and the specific letter represents a form of energy. The letters $m$ and $E$ represent motion energy and electrical energy. The letter $w$ is used to represent wind energy, which the learner represents as distinct from motion energy.

Unlike the previous diagrams, this representation emphasizes the conservation of energy because units of energy are explicitly pictured and the number of units is the same at each instant shown, even as the units change form (as shown by the change in letter) and move to different objects. However, this diagram does not show the tracking of energy. For instance, it is unclear where the two units of energy in the turbine at the third instant came from. It is possible that one $m$ is that same $m$ that was in the turbine at the previous instant; it is also possible that the two units of energy came from the blades or from the air. While this diagram shows the general flow of energy, it does not display the course or trail of a unit of energy.

This diagram includes several other constituent ideas of the NGSS energy model. The form of energy is indicated by
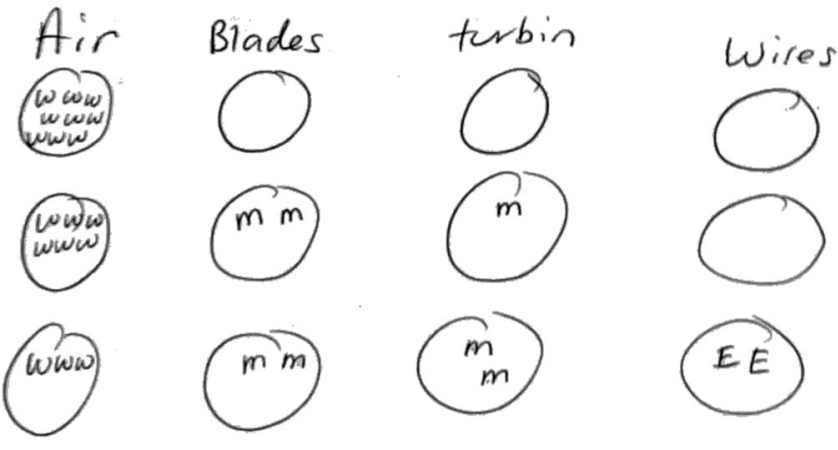

FIG. 8. A learner-invented "energy snapshots" diagram for a wind turbine scenario. the letter of the energy unit and transformations are indicated by a change in letter. Transfers are shown by the energy unit appearing in a different object than in the previous row. The spreading of energy is included since in each successive row, the energy is spread among more objects.

There are several constituent ideas of the NGSS energy model that are not represented in this diagram. This diagram does not show a system, since no boundary is explicitly shown for energy to cross. Mechanisms for energy transfer or transformation are not shown; instead the energy units simply appear in one circle and then another, or change from one letter to another. The diagram also does not show what observables indicate each form of energy.

\section{E. Energy tracking snapshots}

Another learner-invented diagram of the energy in a wind turbine is shown in Fig. 9. This diagram shows the three objects of the scenario (air, turbine, and electrical power system) in the three rows; successive instants of time are shown in the columns of the diagram with time moving from left to right. Individual energy units are labeled using subscripts so a single energy unit can be tracked from one instant in time to the next. For example, energy unit 1 begins in the air as motion energy. It then transfers to the turbine where it transforms to electrical energy. Energy unit 1 then transfers to the electrical power system. Energy unit 2 undergoes the same transfers and transformations as energy unit 1 , at the same times. The other energy units (3 through 6) also experience the same transfers and transformations, but at successively later instants.

This diagram is very similar to the previous diagram. It uses pictures of the energy units in specific objects at successive moments of time (pictured left to right, instead of top to bottom). However, this diagram includes constituent ideas not included in the previous diagram: Because individual units of energy are identified with subscripts that allow a particular energy unit to be followed through each moment in time, it demonstrates both the conservation of energy and the tracking of energy. This diagram includes most of the constituent ideas of the NGSS

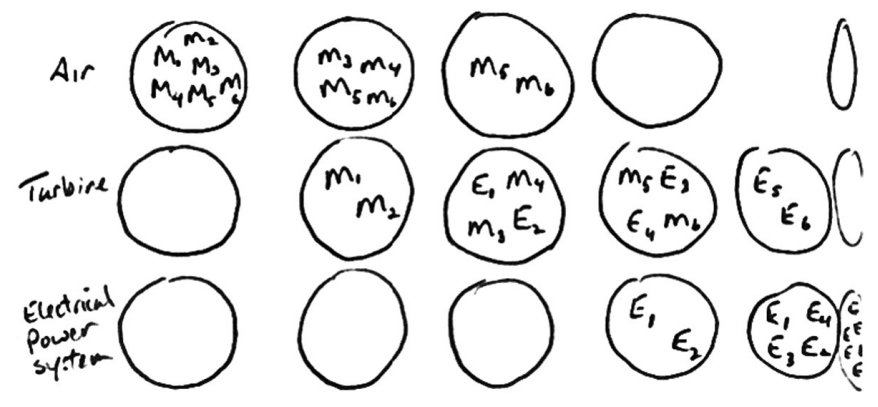

FIG. 9. A learner-invented "energy tracking snapshots" diagram for a wind turbine scenario. 
energy model. The form of energy is indicated by the letter of the energy unit; transformations are indicated by a change in letter with the subscript remaining constant. Transfers are shown by the energy unit appearing in a different row than in the previous column. The spreading of energy is indicated by the energy being distributed among an increasing number of objects.

There are several constituent ideas of the NGSS energy model that are not represented in this diagram. This diagram does not show a system, since no boundary is explicitly shown for energy to cross. Mechanisms for energy transfer or transformation are not shown; instead, the energy units simply appear in one circle then another or change from one letter to another. The diagram also does not show what observables indicate each form of energy.

\section{F. Energy branching}

A learner-invented diagram of a single-bulb circuit is shown in Fig. 10. This diagram shows four circuit elements - the battery, a wire, the bulb filament, and the bulb itself - and the environment around the circuit, labeled "world." Each of these five objects is indicated by a box (the box for the "world" object is $L$ shaped). Units of energy are indicated by letters with the form of the energy unit indicated by the particular letter; arrows from one letter to another indicate the transformation or transfer of that unit of energy and allow energy to be tracked through the scenario. This diagram is very similar to the energy tracking diagram described in Fig. 4, with two important differences. While an energy tracking diagram shows conservation of energy in that each unit of energy can be tracked through the entire scenario, this diagram shows energy units branching into two energy units (in the bottom row of the diagram). Specifically, in the wire the electrical energy unit both transfers to the filament and transforms into thermal energy, which is transferred to the world or environment. In the filament, the thermal energy unit both transforms to light (which is transferred to the bulb) and transfers out into the world. As a result, the scenario begins with three units of energy and ends with five units of energy (found by counting the units at the ends of the chains of arrows), so conservation is violated. Another difference between this learner-invented diagram and an energy

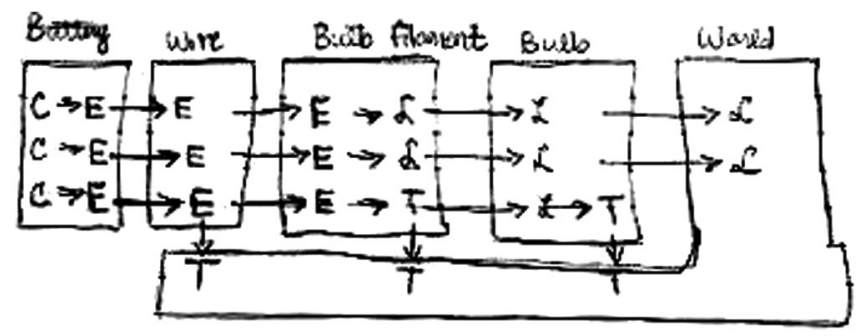

FIG. 10. A learner-invented "energy branching" diagram for a single-bulb circuit scenario. tracking diagram is that this learner-invented diagram does not include the mechanisms for transfers and transformations (the arrows are not labeled). As with energy tracking diagrams, spreading of energy is shown in this diagram by the movement of energy to the world (inferred to be a very large object). As with many of the previous diagrams, systems and observables are not included in this diagram; no boundaries are labeled and there is no indicator of the observable for each form.

\section{INTEREST AND RELIABILITY FOR TEACHERS}

In order to gauge the interest, usefulness, and reliability of the energy diagram checklist for teachers, we asked a group of elementary teachers to use the checklist to evaluate ten sample energy diagrams. These teachers, who had a range of years of teaching experience, had been participating in a year-long professional development program centered on the "Focus on Energy" [47] curriculum for late elementary students. This professional development emphasized understanding the NGSS energy model, developing energy representations, and eliciting and recognizing students' ideas about energy. The energy diagram checklist was not originally part of the professional development course.

Toward the end of the professional development course, one of the authors presented the checklist to the teachers as a possible means to interpret students' energy diagrams. The teachers were given a two-page worksheet that listed each constituent idea along with (i) a definition, (ii) a short bulleted list of how that idea might appear in a diagram, and (iii) another short bulleted list of what would not count towards that idea in a diagram. The teachers then worked with one of the authors (K.G.) to apply the checklist to two sample energy diagrams created by the authors. Approximately 30 minutes of professional development time was spent on this exercise.

Participating teachers spontaneously expressed appreciation for the checklist and stated that it filled a need they had when teaching energy in their classrooms, suggesting that teachers are likely to be interested in the checklist and perceive it as useful. Several of the teachers stated that they intend to use the checklist in their teaching this year. Several chose to use the checklist to analyze the student work that was being discussed in a later professional development activity.

Another purpose of introducing the checklist to teachers was to do a preliminary assessment of reliability with teachers: to observe the extent to which teachers' coding of diagrams agrees with the developers' coding. For this purpose, five of the professional development teachers agreed to code 10 sample energy diagrams as homework, marking which constituent ideas they believed were present in each energy diagram. Five of the sample energy diagrams are teacher-drawn energy diagrams discussed 
above (Figs. 5-9). The other five of the energy diagrams were drawn by elementary students working through the Focus on Energy curriculum.

For each of the nine constituent ideas in each of the ten sample scenarios provided, we documented how many teachers coded that constituent idea as present or absent in that scenario. We then documented how many of the teachers' codes agreed with the developers' codes (for each constituent idea in each scenario). Reliability was assessed as (No. agreements)/(No. possible agreements). With five teachers coding nine constituent ideas in each of ten sample scenarios, the number of possible agreements is $5 \times 9 \times 10=450$. The overall reliability in this exercise (including all constituent ideas in all scenarios) was 0.76 . This represents substantial agreement, given that there was only 30 minutes of training time with no negotiation or practice coding.

The reliability for specific diagrams ranged from 0.60 to 0.95. The diagram shown in Fig. 5 (the "energy picture" of the wind turbine) had the highest reliability, indicating great agreement across all constituent ideas. (In the case of this diagram, this result indicates great agreement that only a few constituent ideas are present in the diagram.) The diagrams with the lowest reliability were the springcompression diagram in Fig. 7 and another diagram using a specialized version of bar charts (not shown): the low reliability of these diagrams may be due to teachers' difficulty interpreting unfamiliar and highly specialized representations.

The reliability for specific constituent ideas ranged from 0.54 to 0.94 . The highest-reliability constituent idea was forms (0.94), followed by conservation (0.88), possibly indicating teachers' deep understanding of and experience recognizing these ideas in a variety of representations. The lowest-reliability constituent idea was tracking (0.54). Tracking is among the greater innovations of the NGSS model of energy (likely less familiar to teachers), and requires an original diagram to adequately represent the concept (see Tables III and IV). While the results of this exercise are preliminary given the small number of teachers involved, they do suggest that teachers are interested in the checklist, perceive it as useful for their teaching, and can reliably apply the checklist to a variety of energy diagrams with minimal training. Future work will expand on this analysis by studying teachers' use of the checklist with their own students and by expanding the group of teachers involved.

\section{ASSESSMENT OF A CLASS'S INCREASED FACILITY WITH ENERGY DIAGRAMS}

The checklist for assessing energy diagrams may be used to assess a class's increased facility with NGSSaligned energy diagrams, i.e., their production of energy diagrams with many NGSS constituent ideas. In 2013, 15 secondary teachers participated in a second-year professional development course focused on the NGSS model of energy [12]. Learning goals included the development and use of NGSS-aligned energy representations such as energy tracking diagrams [12,28]. Participants were given assessments before and after instruction, requesting energy analyses of the ring slider scenario (before), and a steam-turbine power plant (after). The assessments asked them to do the following:

(a) "Draw a diagram showing the energy transfers and transformations within and/or among the objects in the scenario." (For the ring slider, teachers were asked to include the ruler, the ring, the floor, and the surrounding air. For the power plant, they were directed to include the coal in the boiler, the water or steam, the turbine or generator, and the electrical tower.)

(b) "For each transfer and transformation that you indicate in your diagram, describe the mechanism or process by which that transfer or transformation occurred (e.g., metabolism, conduction, compression, melting....)."

Application of the checklist described above shows that the class increased its facility with NGSS-aligned energy diagrams. The results are summarized in Table VII. Before instruction, only one diagram showed conservation of energy; at the end of the course, 12/15 diagrams did. Fewer than half of the learners' diagrams initially included transfers and transformations, whereas at the end of the course all diagrams included these constituent ideas. Initially 5/15 diagrams tracked energy; at the end, $14 / 15$ diagrams did. Mechanisms of energy transfer and transformation were initially indicated in 5/15 diagrams; at the end of the course, 12/15 showed mechanisms. The number of diagrams showing forms of energy went from $9 / 15$ to $15 / 15$, and the number of diagrams showing spreading of energy went from $9 / 15$ to $14 / 15$. No diagrams explicitly indicated a system either before or after instruction; this constituent idea was not among the learning goals for the course. Explicit inclusion of observable indicators of energy decreased from $5 / 15$ to $0 / 15$, probably because

TABLE VII. Class facility with NGSS-aligned energy diagrams $(N=15)$.

\begin{tabular}{lcc}
\hline \hline Constituent idea & Before instruction & After instruction \\
\hline Conservation & 1 & 12 \\
Transfers & 7 & 15 \\
Forms & 9 & 15 \\
Transformation & 6 & 15 \\
Tracking & 5 & 14 \\
Mechanisms & 5 & 12 \\
Spreading $_{\text {System }}^{\text {a }}$ & 9 & 14 \\
Indicators $^{\text {b }}$ & 0 & 0 \\
\hline \hline
\end{tabular}

${ }^{\mathrm{a}}$ Not a learning goal of the course.

${ }^{\mathrm{b}}$ Learners gained facility with a type of diagram that does not typically include this idea. 
learners gained facility with a type of diagram that does not typically include this constituent idea. While this analysis includes a small sample size and considers only one course, this preliminary analysis suggests that the checklist can effectively measure growth and is sensitive to the learning goals of the course.

\section{SUMMARY}

Scientific diagrams and representations support scientists and science learners in communicating their science ideas. Therefore, an important part of science instruction is helping students learn to develop useful scientific representations. Given this goal, teachers need to assess and provide feedback on the effectiveness of students' diagrams for communicating their understanding. A key aspect of a diagram's effectiveness is the extent to which it instantiates the scientific model of the relevant scientific concepts.

Energy is a core idea in the physical, life, and earth, and space sciences, as well as a crosscutting concept of the NGSS. Further, various scientific practices and crosscutting concepts, such as modeling, understanding systems, and cause and effect, are deeply connected to the concept of energy. Teachers look to the Next Generation Science Standards [8] and its parent document, the K12 Framework for Science Education [3], for guidance in both instruction and assessment. However, the model of energy provided by the NGSS is not explicit, especially regarding use or assessment of representations of energy. Thus, our first task in this paper was to elucidate the ideas that the NGSS implies should be included in a model of energy in the physical sciences.

There are multiple commonly used energy diagrams that are intended to communicate different aspects of our understanding of energy. For example, a given diagram may show information about energy values, but not about the processes or mechanisms of energy transfer and transformation. This stands in contrast to teaching about forces, where free body diagrams are accepted and standard representations of the forces acting on an object because the physics community has agreed that they instantiate our model of forces. Our second task in this paper was to propose a checklist that could evaluate in what ways an energy representation instantiates the NGSS model of energy.

To test our checklist, we applied the checklist to three different often-used energy diagrams: bar charts, flow diagrams, and energy tracking diagrams. We found that the bar charts considered show very few aspects of the
NGSS model of energy, while flow diagrams and energy tracking diagrams include more of the model's constituent ideas. Teachers (and students) who are explicitly aware of which constituent ideas are and are not included in a representation are better positioned to evaluate the utility of a given representation for a given instructional context. Some representations may restrict the kinds of learning that are available to students, especially if they do not match instructional goals. For example, a representation that includes no information on energy transfer will not be of great help in classroom discussions of an energy perspective on heat transfer.

In addition to testing our checklist against three established energy representations, we used the checklist to evaluate learner-created energy diagrams. These original diagrams have value in showing students' efforts to synthesize their understanding of energy. Given our goal of designing a checklist to assess the extent to which a representation shows the NGSS model of energy in the physical sciences, we asked whether our checklist can be used to measure a class's increased ability to use representations showing the NGSS model of energy. In a preliminary analysis involving a small group of learners, the answer seems to be yes. Learners' performance on representing energy improved due to a professional development course that emphasized representing nearly all the constituent ideas of energy. Areas that were not addressed in professional development showed no improvement, while areas that were addressed did. This suggests that the checklist is sensitive to the specific activities of instruction and has the correct grain size to accurately reflect and evaluate a learning activity.

\section{ACKNOWLEDGMENTS}

We gratefully acknowledge all the elementary and secondary teachers who have participated in our courses for their generosity in making their reasoning accessible to the research team. We are deeply grateful to these experts in the teaching and learning of energy in physics, for detailed comments on the checklist under development, and assistance with the manuscript: H. G. Close, B. W. Dreyfus, L. A. Elliott, B. W. Harrer, A. D. Robertson, and L. Seeley. Finally, we are grateful to the rest of Seattle Pacific University's Physics Education Research Group, including A. R. Daane, L.S. DeWater, and S.B. McKagan, for substantive discussions of this work. This material is based upon work supported by the National Science Foundation under Grants No. 1418211 and No. 1222777. 
[1] B. Latour, Visualisation and Cognition: Drawing Things Together Knowledge and Society: Studies in the Sociology of Culture Past and Present, edited by H. Kuklick (Jai Press, Greenwich, CT, 1986), Vol. 6, pp. 1-40.

[2] R. Lehrer, L. Schauble, S. Carpenter, and D. Penner, The inter-related development of inscriptions and conceptual understanding, Symbolizing and Communicating in Mathematics Classrooms: Perspectives on Discourse, Tools, and Instructional Design, edited by P. Cobb, E. Yackel, and K. McClain (Routledge, Mahwah, NJ, 2000), pp. 325-60.

[3] National Research Council, A Framework for K-12 Science Education: Practices, Crosscutting Concepts, and Core Ideas (National Research Council, Washington, DC, 2012).

[4] M. Windschitl, J. Thompson, and M. Braaten, Beyond the scientific method: Model-based inquiry as a new paradigm of preference for school science investigations, Sci. Educ. 92, 941 (2008).

[5] G. E. Box, Science and statistics, J. Am. Stat. Assoc. 71, 791 (1976).

[6] D. F. Treagust, G. Chittleborough, and T. L. Mamiala, Students' understanding of the role of scientific models in learning science, Int. J. Sci. Educ. 24, 357 (2002).

[7] R. Devi, A. Tiberghien, M. Baker, and P. Brna, Modelling students' construction of energy models in physics, Instr. Sci. 24, 259 (1996).

[8] NGSS Lead States, Next Generation Science Standards: For States, By States (Washington, DC 2013).

[9] C. V. Schwarz, B. J. Reiser, E. A. Davis, L. Kenyon, A. Achér, D. Fortus, Y. Shwartz, B. Hug, and J. Krajcik, Developing a learning progression for scientific modeling: Making scientific modeling accessible and meaningful for learners, J. Res. Sci. Teach. 46, 632 (2009).

[10] A. Van Heuvelen and X. L. Zou, Multiple representations of work-energy processes, Am. J. Phys. 69, 184 (2001).

[11] E. Brewe, Energy as a substancelike quantity that flows: Theoretical considerations and pedagogical consequences, Phys. Rev. ST Phys. Educ. Res. 7, 020106 (2011).

[12] R. E. Scherr, H. G. Close, S. B. McKagan, and S. Vokos, Representing energy. I. Representing a substance ontology for energy, Phys. Rev. ST Phys. Educ. Res. 8, 020114 (2012).

[13] F. Goldberg, V. K. Otero, and S. Robinson, Physics and Everyday Thinking (It's About Time, Armonk, NY, 2008).

[14] A. Hobson, Energy flow diagrams for teaching physics concepts, Phys. Teach. 42, 113 (2004).

[15] A. Acher, M. Arcà, and N. Sanmartí, Modeling as a teaching learning process for understanding materials: A case study in primary education, Sci. Educ. 91, 398 (2007).

[16] P. Black and D. Wiliam, Assessment and classroom learning, Assess. Educ. 5, 7 (1998).

[17] L. A. Shepard, Commentary: Evaluating the validity of formative and interim assessment, Educ. Meas. Issues Pract. 28, 32 (2009).

[18] D. Wiliam, Keeping learning on track: Classroom assessment and the regulation of learning, Second Handbook of Research on Mathematics Teaching and Learning, edited by F. Lester (Information Age Publishing Greenwich, CT, 2007), pp. 1053-98.
[19] F. Erickson, Some Thoughts on "Proximal" Formative Assessment of Student Learning, Evidence and Decision Making, edited by P. A. Moss (Blackwell Publishing, Malden, MA, 2007), pp. 186-216.

[20] J. Minstrell, R. Anderson, and M. Li, Building on learner thinking: A framework for assessment in instruction (Commissioned paper for the Committee on Highly Successful STEM Schools or Programs for K-12 STEM Education Workshop, 2011).

[21] E. Wenger, Communities of Practice: Learning, Meaning, and Identity (Cambridge University Press, Cambridge, MD, 1998).

[22] L. J. Cronbach and P. L. Meehl, Construct validity in psychological tests, Psychol. Bull. 52, 281 (1955).

[23] J. Loevinger, Objective tests as instruments of psychological theory, Psychol. Rep. 3, 635 (1957).

[24] R. M. Guion, On Trinitarian doctrines of validity, Prof. Psychol. 11, 385 (1980).

[25] A. R. Daane, S. Vokos, and R. E. Scherr, Goals for teacher learning about energy degradation and usefulness, Phys. Rev. ST Phys. Educ. Res. 10, 020111 (2014).

[26] L. Seeley, S. Vokos, and J. Minstrell, Constructing a sustainable foundation for thinking and learning about energy in the twenty-first century, Teaching and Learning of Energy in K-12 Education, edited by R. F. Chen, A. Eisenkraft, D. Fortus, J. S. Krajcik, K. Neumann, J. Nordine, and A. Schef (Springer International Publishing, Switzerland, 2012), pp. 337-56.

[27] B. W. Harrer, V. J. Flood, and M. C. Wittmann, Productive resources in students' ideas about energy: An alternative analysis of Watts' original interview transcripts, Phys. Rev. ST Phys. Educ. Res. 9, 023101 (2013).

[28] R. E. Scherr, H. G. Close, A. R. Daane, L. S. DeWater, B. W. Harrer, A. D. Robertson, L. Seeley, and S. Vokos, Energy tracking diagrams, Phys. Teach. 54, 96 (2016).

[29] A. R. Daane, L. Wells, and R. E. Scherr, Energy theater, Phys. Teach. 52, 291 (2014).

[30] R. E. Scherr, H. Close, E. Close, and S. Vokos, Representing energy. II. Energy tracking representations, Phys. Rev. ST Phys. Educ. Res. 8, 020115 (2012).

[31] A. D. Robertson, R. E. Scherr, L. M. Goodhew, A. R. Daane, K. E. Gray, and L. B. Aker, Identifying content knowledge for teaching energy: Examples from high school physics, Phys. Rev. Phys. Educ. Res. 13, 010105 (2017).

[32] R. E. Scherr and A. D. Robertson, Productivity of "collisions generate heat" for reconciling an energy model with mechanistic reasoning: A case study, Phys. Rev. ST Phys. Educ. Res. 11, 010111 (2015).

[33] H. G. Close and R. E. Scherr, Enacting Conceptual Metaphor through Blending: Learning activities embodying the substance metaphor for energy, Int. J. Sci. Educ. 37, 839 (2015).

[34] R. E. Scherr, H. G. Close, E. W. Close, V. J. Flood, S. B. McKagan, A. D. Robertson, L. Seeley, M. C. Wittmann, and S. Vokos, Negotiating energy dynamics through embodied action in a materially structured environment, Phys. Rev. ST Phys. Educ. Res. 9, 020105 (2013).

[35] A. R. Daane, S. B. McKagan, S. Vokos, and R. E. Scherr, Energy conservation in dissipative processes: Teacher expectations and strategies associated with imperceptible 
thermal energy, Phys. Rev. ST Phys. Educ. Res. 11, 010109 (2015).

[36] T. G. Amin, Conceptual metaphor meets conceptual change, Hum. Dev. 52, 165 (2009).

[37] A. A. diSessa, Ontologies in pieces: Response to Chi and Slotta, Cognit. Instr. 10, 272 (1993).

[38] R. Duit, Should energy be illustrated as something quasimaterial?, Int. J. Sci. Educ. 9, 139 (1987).

[39] G. Falk, F. Herrmann, and G. Bruno Schmid, Energy forms or energy carriers?, Am. J. Phys. 51, 1074 (1983).

[40] R. Millar, Teaching about Energy (Department of Educational Studies, University of York, York, UK, 2005).

[41] G. Swackhamer, Cognitive resources for understanding energy, http://modeling.asu.edu/modeling/CognitiveResourcesEnergy.pdf.

[42] B. W. Harrer, On the origin of energy: Metaphors and manifestations as resources for conceptualizing and measuring the invisible, imponderable, Am. J. Phys. 85, 454 (2017).

[43] B. W. Dreyfus, B. D. Geller, J. Gouvea, V. Sawtelle, C. Turpen, and E.F. Redish, Ontological metaphors for negative energy in an interdisciplinary context, Phys. Rev. ST Phys. Educ. Res. 10, 020108 (2014).

[44] H. Jin and C. W. Anderson, A learning progression for energy in socio-ecological systems, J. Res. Sci. Teach. 49, 1149 (2012).

[45] Khan Academy Physics, LOL diagrams Work and energy I Physics I Khan Academy-YouTube, www.youtube.com/ watch? $=$ =_8RohfPMklM (2016).

[46] C. Kittel and H. Kroemer, Thermodynamics (Freeman \& Co. New York City, 1980).

[47] TERC 2015, Focus on Energy, https://focusonenergy.terc .edu/. 